$\mathbf{T}_{\text {he }} \mathbf{J}_{\text {ournal of }} \mathbf{N}_{\text {onlinear }} \mathbf{S}_{\text {ciences and }}$ Applications

http://www.tjnsa.com

\title{
EXACT CONTROLLABILITY OF SEMILINEAR THIRD ORDER DISPERSION EQUATION
}

\author{
N.K. TOMAR ${ }^{1 *}$ AND N. SUKAVANAM ${ }^{2}$
}

\begin{abstract}
In this paper, a family of nonlinear functions is given for the exact controllability of semilinear third order dispersion equation. The obtained result has been illustrated by applying it on nonlinear Korteweg-de Vries (KdV) equation.
\end{abstract}

\section{INTRODUCTION}

Let us consider the semilinear third-order dispersion equation of the form

$$
\frac{\partial \omega}{\partial t}(x, t)+\frac{\partial^{3} \omega}{\partial x^{3}}(x, t)=(G u)(x, t)+f(t, \omega(x, t))
$$

in the domain $0 \leq x \leq 2 \pi, t \geq 0$, such that the process is periodic with period $2 \pi$ in the variable $x$ with boundary conditions

$$
\frac{\partial^{k} \omega}{\partial x^{k}}(0, t)=\frac{\partial^{k} \omega}{\partial x^{k}}(2 \pi, t) ; k=0,1,2
$$

and initial condition

$$
\omega(x, 0)=0,
$$

where $u$ is the control function and $f:[0, \infty) \times \mathbb{R} \rightarrow \mathbb{R}$ is a nonlinear continuous function.

By taking $G=0$ (i.e. without the control term) and nonlinear function $f(t, \omega)=$ $-\omega_{x}-\omega \omega_{x}$, the equation (1.1) reduces into the partial differential equation model for the famous Korteweg-de Vries (KdV) equation (for derivation and more details see [1]), which describes the propagation of small amplitude long water waves in

Date: Received: Jun 21, 2011; Revised: December 12, 2011.

${ }^{*}$ Corresponding author

(c) 2011 N.A.G.

2000 Mathematics Subject Classification. Primary 93C20; Secondary 92B20, 90C90.

Key words and phrases. Exact controllability, Dispersion System, KdV Equation. 
a uniform channel, and in this context, $\omega$ is the amplitude or velocity, $x$ is often proportional to distance in the direction of propagation and $t$ is proportional to elapsed time. There is a vast amount of literature on the controllability of linear $\mathrm{KdV}$ equation with the controls either as distributed controls or through the boundary, see $[2,3,4,5]$ and references therein. The study of controllability of system (1.1)-(1.3) is important because this system is not only a good model for water waves but also a very useful model in nonlinear studies in many areas of engineering and science like laser theory, observations of the solitary waves, study of solitons in hydrodynamics and plasma theory etc. (see $[6,7,8]$ and references therein). The controllability of an abstract semilinear control system in infinite dimensional spaces is an important property and has been studied by many authors in recent past via functional analysis approach, see $[9,10,11,12$, $13]$.

For the sake of brevity, we shall write $V$ and $Z$ for the spaces $L_{2}(0,2 \pi)$ and $L_{2}\left[0, \tau ; L_{2}(0,2 \pi)\right]$ respectively. In (1.1), the state $\omega(., t)$ takes values in the Banach space $V$ and the control function $u \in Z$.

From the practical point of view, we restrict the distributed control $u(x, t)$ so that the quantity $[\omega]=\int_{0}^{2 \pi} \omega(x, t) d x$ is conserved, and in order to conserve this quantity, we define the bounded linear operator $G$ on $Z$ as

$$
(G u)(x, t)=g(x)\left\{u(x, t)-\int_{0}^{2 \pi} g(s) u(s, t) \mathrm{d} s\right\}
$$

where $g(x)$ is a piece-wise continuous positive function on $[0,2 \pi]$ such that (see [14])

$$
[g]=\int_{0}^{2 \pi} g(s) \mathrm{d} s=1 .
$$

In [14], Russell and Zhang proved the controllability and stabilization of the third order linear dispersion equation. They discussed the exponential decay rates with distributed controls of restricted form and for the equation with boundary dissipation. George et. al. [15] proved the exact controllability of (1.1)-(1.5), in the case when $f$ is a Lipschitz continuous function with sufficiently small Lipschitz constant.

\section{Preliminaries and Hypotheses}

The purpose of this paper is to study the exact controllability of the semilinear third order dispersion system (1.1)-(1.5).

Define an operator $A$ on $V$ with domain $D(A) \subseteq H^{3}(0,2 \pi)$ consisting of functions satisfying boundary conditions of the form (1.2) such that

$$
A \omega=-\frac{\partial^{3} \omega}{\partial x^{3}}
$$

It follow from Pazy [16] that $A$ generates a $C_{0}$ group of isometries, $\{T(t)\}_{t \geq 0}$, on space $V$. By the variation of constant formula, we can write a mild solution of 
(1.1)-(1.3) as

$$
\omega(\cdot, t)=\int_{0}^{t} T(t-s)(G u)(\cdot, s) \mathrm{d} s+\int_{0}^{t} T(t-s) f(s, \omega(\cdot, s)) \mathrm{d} s
$$

Remark 2.1. Note that, some sufficient conditions are available in the literature for the existence of the mild solutions of system of the form (1.1)-(1.3), see [15, 16]. In the present paper we will show the exact controllability of the system (1.1)(1.3) whose mild solution exists.

Let $V_{1}$ be a subspace of $V$ defined as

$$
V_{1}=\left\{x \in V:\left[x_{T}\right]=\int_{0}^{2 \pi} x(s) \mathrm{d} s=0\right\} .
$$

It is proved in [15] that $V_{1}$ is a Hilbert space with respect to $L_{2}$-norm.

Let us analyze the exact controllability property:

Definition 2.2. The system (1.1)-(1.5) is said to be exactly controllable over a time interval $[0, \tau]$, if for any given $\omega_{\tau} \in V_{1}$ there exists a control $u \in Z$ such that the corresponding solution $\omega$ of $(1.1)-(1.5)$ satisfies $\omega(\cdot, \tau)=\omega_{\tau}$.

In this paper we investigated a family of nonlinear functions $f(t, \omega(x, t))=$ $f_{1}(t, \omega(x, t))+f_{2}(t, \omega(x, t))+f_{3}(t, \omega(x, t))$ for that the system (1.1)-(1.5) is exact controllable, when

(H1) $R\left(F_{1}\right) \subseteq N(L)$,

(H2) $\int_{0}^{2 \pi} f_{2}(t, \omega(x, t)) \mathrm{d} x=0$,

(H3) $f_{3}$ is Lipschitz continuous with sufficiently small Lipschitz constant, i.e., it satisfies the following inequality,

$$
\left|f_{3}(t, x)-f_{3}(t, y)\right| \leq l|x-y| ; \forall x, y \in \mathbb{R} \text { and } \mathrm{t} \in[0, \tau]
$$

with small constant $l$.

where operators $L: Z \rightarrow V_{1}$ and $F_{1}: Z \rightarrow Z$ are defined as

$$
\begin{gathered}
\left.L \omega=\int_{0}^{\tau} T(\tau-s) \omega(\cdot, s)\right) \mathrm{d} s \\
\left(F_{1} \omega\right)(t)=f_{1}(t, \omega(\cdot, t)) .
\end{gathered}
$$

\section{Main Result}

Before proving the main theorem, we shall prove the following lemma

Lemma 3.1. If $f_{2}$ satisfies the condition (H2), then for each $\omega \in Z$ there exists $u \in Z$ such that, for any fixed $t$

$$
(G u)(x, t)=f_{2}(t, \omega(x, t)) .
$$

Proof. Eq. (3.1) is equivalent to the following integral equation

$$
g(x)\left\{u(x, t)-\int_{0}^{2 \pi} g(s) u(s, t) \mathrm{d} s\right\}=f_{2}(t, \omega(x, t))
$$


or

$$
u(x, t)-\int_{0}^{2 \pi} g(s) u(s, t) \mathrm{d} s=\frac{f_{2}(t, \omega(x, t))}{g(x)}
$$

Now, we define operator $H: L_{2}(0,2 \pi) \rightarrow L_{2}(0,2 \pi)$ as

$$
(H u)(x)=u(x)-\int_{0}^{2 \pi} g(s) u(s) \mathrm{d} s
$$

It is easy to see that the null space of operator $H$, i.e. $N(H)$, is one dimensional subspace of $L_{2}(0,2 \pi)$ and is spanned by the set $\{1\}$. Now we shall find the operator $H^{*}$ as follows:

Let $v \in L_{2}(0,2 \pi)$, then

$$
\begin{aligned}
\langle H u, v\rangle_{L_{2}(0,2 \pi)} & =\int_{0}^{2 \pi}(H u)(y) v(y) \mathrm{d} y \\
& =\int_{0}^{2 \pi} u(y) v(y) \mathrm{d} y-\int_{0}^{2 \pi}\left(\int_{0}^{2 \pi} g(s) u(s) d s\right) v(y) \mathrm{d} y \\
& =\langle u, v\rangle_{L_{2}(0,2 \pi)}-\int_{0}^{2 \pi} \mathrm{d} s u(s)\left(\int_{0}^{2 \pi} g(s) v(y) \mathrm{d} y\right) \\
& =\left\langle u, H^{*} v\right\rangle_{L_{2}(0,2 \pi)}
\end{aligned}
$$

Thus,

$$
\begin{aligned}
\left(H^{*} v\right)(s) & =v(s)-\int_{0}^{2 \pi} g(s) v(y) \mathrm{d} y \\
& =v(s)-\alpha g(s)
\end{aligned}
$$

where $\alpha=\int_{0}^{2 \pi} v(y) \mathrm{d} y$. Hence, the null space of operator $H^{*}$, i.e. $N\left(H^{*}\right)$, is the space spanned by the set $\{g(\cdot)\}$.

It is clear that $H$ is a fredholm operator, it follows that (see [17])

$$
R(H)=N\left(H^{*}\right)^{\perp}
$$

Hence (3.3) has a solution $u(\cdot, t) \in L_{2}(0,2 \pi)$ if

$$
\left\langle\frac{f_{2}(t, \omega(\cdot, t))}{g(\cdot)}, \alpha g(\cdot)\right\rangle_{L_{2}(0,2 \pi)}=0,
$$

that is, if

$$
\int_{0}^{2 \pi} f_{2}(t, \omega(x, t)) \mathrm{d} x=0
$$

Eq. (3.8) concludes that if condition (H2) holds true, eq. (3.3) has a solution $u(\cdot, t) \in L_{2}(0,2 \pi)$ for any fixed $t \in[0, \tau]$.

Theorem 3.2. [Main Theorem] Suppose that the nonlinear function $f$ in Eq. (1.1) is expressed as $f(t, \omega(x, t))=f_{1}(t, \omega(x, t))+f_{2}(t, \omega(x, t))+f_{3}(t, \omega(x, t))$, where $f_{1}, f_{2}$ and $f_{3}$ satisfy assumptions (H1), (H2) and (H3) respectively, then the system (1.1)-(1.5) is exact controllable on $V_{1}$. 
Proof. Let $v \in V_{1}$. Consider the following semilinear system (with the same conditions as given in (1.2)-(1.3))

$$
\frac{\partial \omega}{\partial t}(x, t)+\frac{\partial^{3} \omega}{\partial x^{3}}(x, t)=(G u)(x, t)+f_{3}(t, \omega(x, t))
$$

From Theorem 4.1 in [15], it follows that above system is controllable, i.e., there exists a $u \in Z$ such that

$$
v=\int_{0}^{\tau} T(\tau-s)(G u)(\cdot, s) \mathrm{d} s+\int_{0}^{\tau} T(\tau-s) f_{3}(s, \omega(\cdot, s)) \mathrm{d} s .
$$

By condition (H1)

$$
\int_{0}^{\tau} T(\tau-s) f_{1}(s, \omega(\cdot, s)) \mathrm{d} s=0
$$

By adding equations (3.10) and (3.11) we get

$$
\begin{aligned}
v=\int_{0}^{\tau} T(\tau-s)(G u)(\cdot, s) \mathrm{d} s & +\int_{0}^{\tau} T(\tau-s) f_{1}(s, \omega(\cdot, s)) \mathrm{d} s \\
& +\int_{0}^{\tau} T(\tau-s) f_{3}(s, \omega(\cdot, s)) \mathrm{d} s
\end{aligned}
$$

By Lemma 3.1, we can find a $u_{1} \in Z$ such that for all $t \in[0, \tau]$

$$
\left(G u_{1}\right)(\cdot, t)=f_{2}(t, \omega(\cdot, t)) .
$$

Using equations (3.12) and (3.13) we conclude that

$$
\begin{aligned}
v & =\int_{0}^{\tau} T(\tau-s)\left(G u-u_{1}\right)(\cdot, s) \mathrm{d} s+\int_{0}^{\tau} T(\tau-s) f_{1}(s, \omega(\cdot, s)) \mathrm{d} s \\
& +\int_{0}^{\tau} T(\tau-s) f_{2}(s, \omega(\cdot, s)) \mathrm{d} s+\int_{0}^{\tau} T(\tau-s) f_{3}(s, \omega(\cdot, s)) \mathrm{d} s
\end{aligned}
$$

Eq. (3.14) implies that $v$ is a solution of the system (1.1)-(1.5) with respect to control $u-u_{1}$. This proves the exact controllability of (1.1)-(1.5). Hence, the proof is complete.

Remark 3.3. Since $f_{1} \equiv 0$ and $f_{2} \equiv 0$ satisfies the conditions (H1) and (H2) respectively, the Theorem 4.1 of [15] follows as a particular case of above theorem.

Remark 3.4. If $f_{1} \equiv 0$ and $f_{3} \equiv 0$, conditions (H1) and (H3) are satisfied automatically. Now, take $f_{2}(t, \omega(x, t))=-\omega(x, t) \omega_{x}(x, t)$, then problem (1.1)-(1.5) is converted into the exact controllability of nonlinear $\mathrm{KdV}$ equation. In this case,

the condition $(\mathrm{H} 2), \int_{0}^{2 \pi} f_{2}(t, \omega(x, t)) \mathrm{d} x=0$, is also satisfied. Thus, Theorem 3.2 gives the exact controllability of nonlinear $\mathrm{KdV}$ equation, which support the result of Theorem 1.1 of Russell and Zhang [2].

\section{CONCLUSiOn}

In this work, a family of nonlinear functions $f=f_{1}+f_{2}+f_{3}$ has been given, where $f_{1}, f_{2}, f_{3}$ satisfy the conditions (H1), (H2), (H3) respectively, for the exact controllability of system (1.1)-(1.5). Thus, Theorem 3.2 gives more choices of nonlinear functions than the paper [15], in which authors proved the exact controllability of system (1.1)-(1.5) by taking $f=f_{3}$, under the assumption 
(H3). In [15], the imposed condition (H3) force us to consider the semilinear system (1.1)-(1.5) as a nonlinear perturbation of corresponding linear system. The exact controllability of nonlinear general KdV equation has also been given, as a consequence of Theorem 3.2, in Remark 3.4.

\section{REFERENCES}

1. D. J. Korteweg and G. de Vries, On the change of the form of long waves advancing in a rectangular canal, and on a new type of long stationary waves, Philos. Mag. 39 (1895), 422-443. 1

2. D. L. Russell and B. Y. Zhang, Exact Controllability and Stabilizability of the Korteweg-de Vries Equation, Trans. Amer. Math. Soc. 348(9) (1996), 3643-3672. 1, 3.4

3. L. Rosier, Exact Boundary Controllability for the Korteweg-de Vries Equation on a Bounded Domain, ESIAM Control Optim. Calc. Var. 2 (1997), 33-55. 1

4. B. Y. Zhang, Exact Controllability for the Korteweg-de Vries Equation, SIAM J. Control Optim. 37(2) (1999), 543-565. 1

5. L. Rosier, Exact Boundary Controllability for the linear Korteweg-de Vries Equation on the half line, SIAM J. Control Optim. 39(2) (2000), 331-351. 1

6. K. Hizanidis, B. A Malomed, H. E Nistazakis. and D. J Frantzeskakis, Stabilizing soliton transmission by third-order dispersion in dispersion-compensated fibre links, Pure Appl. Opt. 7(4) (July 1998), L57-L62. 1

7. Yushu Liu, Time Jitters caused by Third-Order Dispersion in Soliton Transmission System, Int. J. Infrared and Millimeter Waves 20(8) (1999), 1541-1548. 1

8. N. Kkelil, N. Bensalah N. and A. Zerarka, Artificial Perturbation for Solving Korteweg-de Vries Equation, J. Zhejiang Univ. Sci. A 7(12) (2006), 2079-2082. 1

9. Balachandran K. and Dauer J.P. Controllability of Nonlinear Systems in Banach Spaces: A Survey. J. Optimization Theory and Applications 115(1) (2002) 7-28. 1

10. N. K. Tomar and N. Sukavanam, Approximate Controllability of Non-densely defined Semilinear Delayed Control Systems, Nonlinear Studies, 18(2) (2011), 229-234. 1

11. J. P. Dauer and N. I. Mahmudov, Approximate Controllability of Semilinear Functional Equations in Hilbert Spaces, J. Math. Anal. Appl, 273 (2002) 310-327. 1

12. Jeong J.-M. and Kim H.-G, Controllability for semilinear functional integrodifferential equations, Bull. Korean Math. Soc., 46(3) (2009) 463-475. 1

13. V Kavitha V and M. Mallika Arjunan, Controllability of impulsive Quasi-linear Fractional mixed volterra-Fredholm-type integrodifferential equations in Banach Spaces, J. Nonlineare Sci. Appl., 4(2) (2011) 152-159. 1

14. D. L. Russell and B. Y. Zhang, Controllability and Stabilizability of the Third-order Linear Dispersion Equation on a Periodic Domain, SIAM J. Control Optim. 31(3) (1993), 659676. 1,1

15. R. K. George, D. N. Chalishajar and A. K. Nandakumaran, Exact Controllability of the Nonlinear Third-order Dispersion Equation, J. Math. Anal. Appl. 332 (2007), 1028-1044. $1,2.1,2,3,3.3,3$

16. A. Pazy, Semigroup of Linear operators and Application to Partial Differential Equations, Springer-Verlag New York Inc., 1983. 2, 2.1

17. E. Zeidler, Applied Fuctional Analysis: Main Principles and Their Applications, Applied Mathematical Sciences Vol. 109, Springer-Verlag New York Inc., 1995. 3

${ }^{1}$ Department of Mathematics, Indian Institute of Technology Patna-800013, INDIA.

E-mail address: nktomar@iitp.ac.ir 
2 Department of Mathematics, Indian Institute of Technology Roorkee-247667, INDIA.

E-mail address: nsukvfma@iitr.ernet.ir 